\title{
Prolonged red cell storage before transfusion increases extravascular hemolysis
}

\author{
Francesca Rapido, ${ }^{1,2}$ Gary M. Brittenham, ${ }^{3,4}$ Sheila Bandyopadhyay, ${ }^{1}$ Francesca La Carpia, ${ }^{1}$ Camilla L'Acqua, ${ }^{1}$ Donald J. McMahon, ${ }^{4}$ \\ Abdelhadi Rebbaa, ${ }^{1}$ Boguslaw S. Wojczyk, ${ }^{1}$ Jane Netterwald, ${ }^{1}$ Hangli Wang, ${ }^{1}$ Joseph Schwartz, ${ }^{1}$ Andrew Eisenberger, ${ }^{4}$ \\ Mark Soffing, ${ }^{5}$ Randy Yeh, ${ }^{5}$ Chaitanya Divgi, ${ }^{5}$ Yelena Z. Ginzburg, ${ }^{6}$ Beth H. Shaz, ${ }^{6}$ Sujit Sheth, ${ }^{7}$ Richard 0. Francis, \\ Steven L. Spitalnik, ${ }^{1}$ and Eldad A. Hod ${ }^{1}$ \\ 'Department of Pathology and Cell Biology, Columbia University College of Physicians and Surgeons - New York Presbyterian Hospital, New York, New York USA. ${ }^{2}$ Department of Medical-Surgical \\ Pathophysiology and Organ Transplantation, Università degli Studi di Milano, Milan, Italy. ${ }^{3}$ Departments of Pediatrics, ${ }^{4}$ Medicine, and ${ }^{5}$ Nuclear Medicine, Columbia University College of Physicians and \\ Surgeons - New York Presbyterian Hospital, New York, New York USA. ${ }^{6}$ New York Blood Center, New York, New York, USA. 'Department of Pediatrics, Weill Cornell Medical College - New York Presbyterian \\ Hospital, New York, New York USA.
}

\begin{abstract}
BACKGROUND. Some countries have limited the maximum allowable storage duration for red cells to 5 weeks before transfusion. In the US, red blood cells can be stored for up to 6 weeks, but randomized trials have not assessed the effects of this final week of storage on clinical outcomes.
\end{abstract}

\begin{abstract}
METHODS. Sixty healthy adult volunteers were randomized to a single standard, autologous, leukoreduced, packed red cell transfusion after $1,2,3,4,5$, or 6 weeks of storage ( $n=10$ per group). 51-Chromium posttransfusion red cell recovery studies were performed and laboratory parameters measured before and at defined times after transfusion.

RESULTS. Extravascular hemolysis after transfusion progressively increased with increasing storage time $(P<0.001$ for linear trend in the AUC of serum indirect bilirubin and iron levels). Longer storage duration was associated with decreasing posttransfusion red cell recovery $(P=0.002)$, decreasing elevations in hematocrit $(P=0.02)$, and increasing serum ferritin $(P<0.0001)$. After 6 weeks of refrigerated storage, transfusion was followed by increases in AUC for serum iron $(P<0.01)$, transferrin saturation $(P<0.001)$, and nontransferrin-bound iron $(P<0.001)$ as compared with transfusion after 1 to 5 weeks of storage.
\end{abstract}

CONCLUSIONS. After 6 weeks of refrigerated storage, transfusion of autologous red cells to healthy human volunteers increased extravascular hemolysis, saturated serum transferrin, and produced circulating nontransferrin-bound iron. These outcomes, associated with increased risks of harm, provide evidence that the maximal allowable red cell storage duration should be reduced to the minimum sustainable by the blood supply, with 35 days as an attainable goal.

REGISTRATION. ClinicalTrials.gov NCT02087514.

FUNDING. NIH grant HL115557 and UL1 TR000040.

\section{Introduction}

Red blood cell transfusion, the most common procedure performed on hospitalized patients (1), is an indispensable component of modern medicine. Establishing an adequate blood supply depends on the ability to store donated red cells safely. The US FDA allows refrigerated storage of red cells for up to 42 days before transfusion. The FDA-approved red cell storage duration is not based on evidence of clinical safety or effectiveness, but was derived from stan-

\section{Related Commentary: p. 100}

Conflict of interest: The authors have declared that no conflict of interest exists. Role of funding source: The sponsor was not involved in the study design, collection, analysis, and interpretation of data, writing of the manuscript, or decision to submit the manuscript for publication.

Submitted: September 21, 2016; Accepted: October 27, 2016

Reference information: J Clin Invest. 2017;127(1):375-382. doi:10.1172/JCI90837. dards set before the advent of clinical outcome studies (2). During refrigeration, red cells undergo multiple physiologic changes, collectively called the red cell "storage lesion" (3). The storage duration that produces a storage lesion sufficiently severe to increase transfusion-related morbidity or mortality is unknown. Furthermore, no identified components of the storage lesion reliably predict the clinical consequences of transfusing an individual red cell unit. After animal and observational human studies suggested that transfusions of older, refrigerator storage-damaged red cells were associated with increased morbidity and mortality (4), several randomized controlled trials compared transfusion of "fresher" with "standard practice" or "older" red cells (4-8). None of these trials found clinically significant outcome differences when comparing transfusions of red cells stored for shorter $(\sim 1$ week) or longer $(\sim 2$ to 5 week) periods. Critically, neither these trials nor others now in progress specifically examine the risks associated with transfusing red cells after 35 to 42 days of storage (4). In the US, approximately 


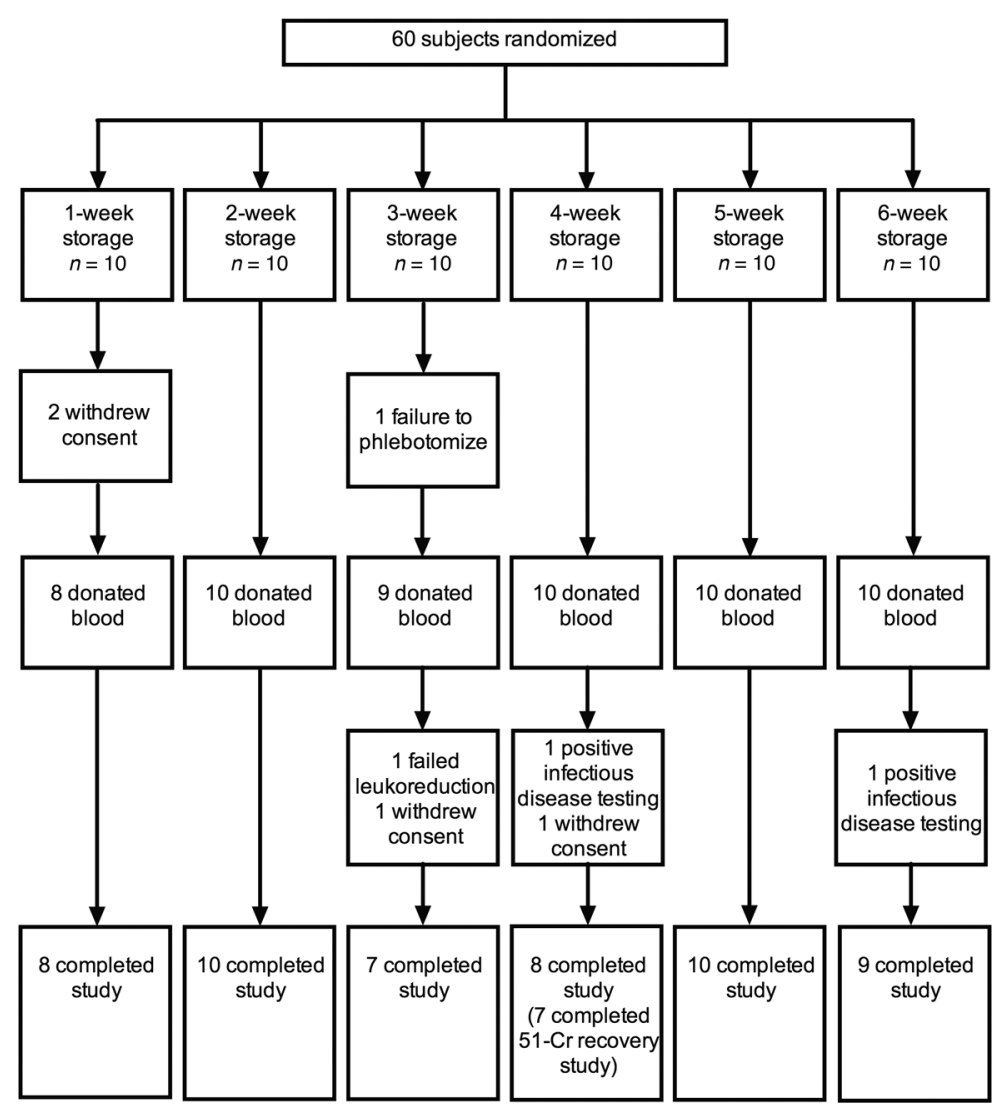

Figure 1. Number of subjects who were randomized, dropped out, and completed the study. 51-Cr, 51-chromium.

14 million units of whole blood and red cells are collected annually (9). The National Heart, Lung and Blood Institute Recipient Epidemiology and Donor Evaluation Study III (REDS-III) found that $9.7 \%-20.7 \%$ of red cell units transfused at 7 hospitals were stored for longer than 35 days (10); thus, a considerable number of patients are potentially at risk.

Concerns about potential harm from transfusing the oldest blood have led the United Kingdom, Ireland, the Netherlands, and large blood services in Germany to restrict the maximum red cell storage duration to 35 days (11); the US NIH Blood Bank has a similar policy (12). A retrospective review of 28,247 transfused patients provided new evidence that transfusing red cells near their 42-day storage limit may have harmful effects (13). This study compared clinical outcomes in patients transfused exclusively with red cells stored not more than 21 days with those in patients transfused exclusively with red cells stored 35 days or more. In critically ill patients, red cells stored for 35-42 days were associated with increased morbidity $(P=0.002)$ and mortality $(P=0.009)(13)$. Although prospective data are needed to guide clinical practice, prospective clinical trials cannot determine the storage duration that increases the risk of harmful events because, for ethical reasons, patients cannot be randomly assigned to receive the oldest blood (12). As an alternative, we randomized healthy adults to a single standard, autologous, leukoreduced, packed red cell transfusion after 1, 2, 3, 4,5 , or 6 weeks of storage, determined 51-chromium 20-hour red cell recoveries, and measured laboratory indicators of hemolysis and iron homeostasis. Our primary outcome was the appearance of circulating nontransferrin-bound iron, indicating that the physiologic capacity to process the iron released from the catabolism of cleared, refrigerated storage-damaged red cells was exceeded.

\section{Results}

Study participant demographics and recruitment. All outcomes were collected between March 24, 2014, and January 20, 2016. Of the 60 subjects randomized, 52 completed the study and had evaluable primary outcomes (Figure 1); one of these subjects did not complete the posttransfusion recovery study for scheduling reasons. The primary reasons for unsuccessful completion included personal reasons $(n=4)$, blood donation technical failures $(n=2)$, and positive infectious disease testing $(n=2)$. The groups had similar baseline characteristics (Table 1$)$.

Circulating nontransferrin-bound iron is produced in transfusion recipients after 6 weeks of red cell storage. Following transfusions of 1 autologous, packed red cell unit, the amount of iron entering the circulation exceeded the iron-uptake capacity of transferrin (the physiologic iron transport protein), producing circulating nontransferrin-bound iron in 10\% (1/10) and 78\% (7/9) of subjects transfused with red cells stored for 5 and 6 weeks, respectively $(P=0.003)$ (Figure $2 A)$. The AUC of the change in nontransferrin-bound iron after 6 weeks of storage was increased, as compared with all other groups $(P<0.001)$ (Figure $2 \mathrm{~B})$.

Transfusion of red cells after longer storage duration induces increased extravascular hemolysis, but not intravascular hemolysis. Following transfusion, serum indirect bilirubin levels progressively increased with increasing storage time $(P<0.001$ for linear trend in AUC; Figure 3A). The AUC of the change in indirect bilirubin differed significantly when comparing subjects randomized to 6-week-stored red cells relative to 1- and 2-weekstored red cells $(P=0.003$ and $P=0.004$, respectively). Concurrently, posttransfusion serum iron concentration progressively increased with increasing storage time $(P<0.0001$ for linear trend in AUC; Figure 3B). The AUC of the change in serum iron after 6 weeks of red cell storage was increased, as compared with all other groups $(P<0.01$ for all comparisons). Furthermore, all subjects transfused with 6-week-stored red cells had increased serum iron above the upper limit of our clinical laboratory's healthy reference range (i.e., $158 \mu \mathrm{g} / \mathrm{dl}$ ). Consistent with increasing serum iron, transferrin saturation increased with increasing storage time $(P<0.0001$ for linear trend in AUC; Figure 3C). The AUC of the change in transferrin saturation was significantly different in subjects randomized to 6-week-stored red cells as compared with all other groups $(P<0.001$ for all comparisons $)$ and with subjects randomized to 5 -week-stored red cells relative to 1 and 2 weeks of storage $(P=0.007$ and $P=0.04$, respectively).

Lactate dehydrogenase (Figure 4A), haptoglobin (Figure $4 \mathrm{~B}$ ), and plasma-free hemoglobin (Figure 4C) levels did not differ among the groups. Although decreases in haptoglobin were observed in all groups, the decreases were parallel to concurrent 
Table 1. Demographic characteristics of subjects completing the study

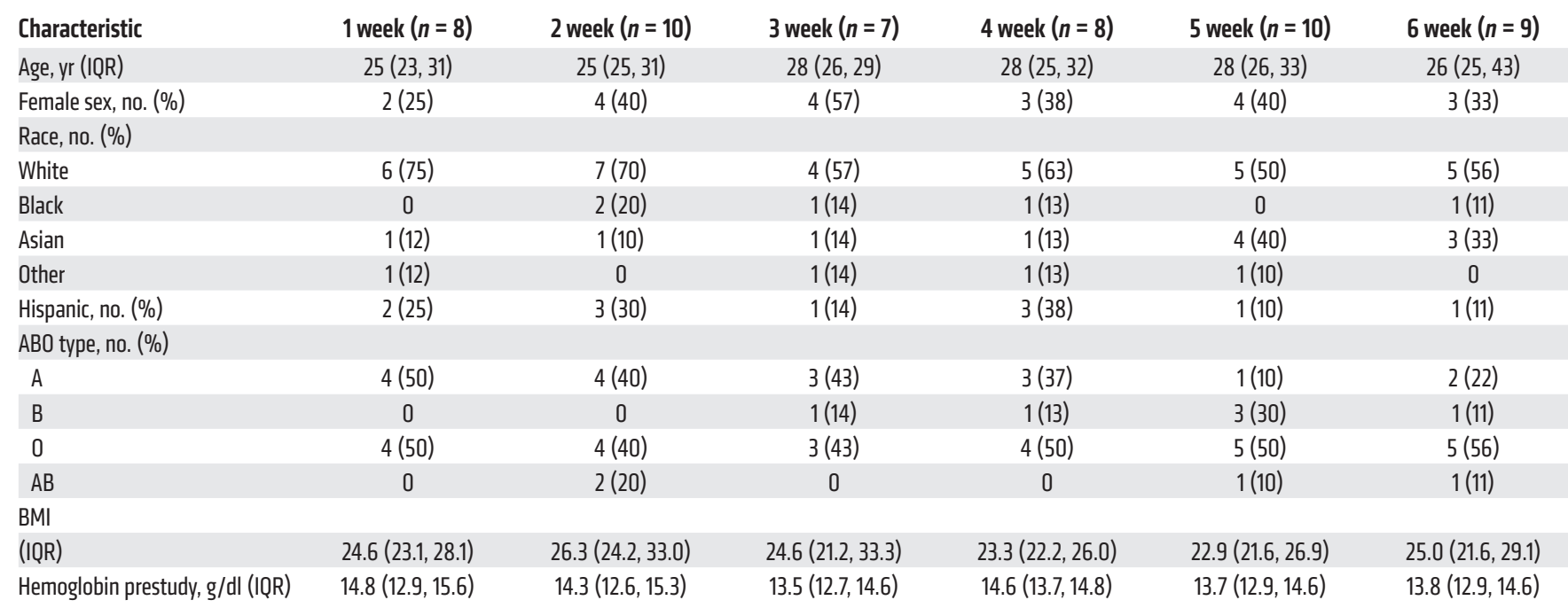

Race/ethnicity was self-reported by subjects. IQR, interquartile range.

decreases in total serum protein from immediately after transfusion to 20 hours after transfusion (Figure 4D). The changes in total serum protein also did not differ between the groups.

Transfusion of red cells after longer duration of storage induces increasing hepcidin, but not IL-6, levels. Hepcidin, the master regulator of iron homeostasis, is regulated by iron and inflammation (14). Following transfusion, serum hepcidin levels progressively increased with increasing storage time $(P=0.005$ for linear trend in AUC; Figure 5A). The AUC of the change in hepcidin differed significantly when comparing subjects randomized to 6-week-stored red cells relative to 1- and 2-week-stored red cells ( $P=0.004$ and $P=0.049$, respectively). There was not a temporally associated rise in IL-6 levels, and IL-6 levels did not differ between the groups (Figure 5B).

Measures of red cell clearance are increased after transfusion of red cells stored for longer duration. Only 1 of the 52 study subjects had a 20-hour posttransfusion red cell recovery below the mean FDA guideline of $75 \%$ (Figure 6A). Furthermore, the percentage of posttransfusion red cell recovery (i.e., the percentage of transfused red cells surviving in circulation for at least 20 hours) decreased with increasing storage time (Pearson coefficient $r=0.42, P=0.002$ ). Similarly, the change in hematocrit, from pretransfusion to 20 hours after transfusion, decreased with increasing storage duration (Pearson coefficient $r=0.32, P=0.02$ ) (Figure 6B). Finally, the change in ferritin, from pretransfusion to 20 hours after transfusion, increased with increasing storage duration (Pearson coefficient $r=0.52, P<0.0001$ ) (Figure 6C).

There were no adverse effects attributable to the transfusions in healthy volunteers. Only 1 adverse event was reported to the investigators and the Data and Safety Monitoring Board (DSMB) at Columbia University Medical Center. This subject, randomized to transfusion with 5 -week-stored red cells, developed a tooth abscess 2 days after transfusion, but had a prior history of poor dentition and abscesses; thus, the subject and DSMB deemed it unlikely to be associated with transfusion. Vital signs also remained stable in all subjects throughout the study, with no clinically significant differences in temperature, heart rate, or blood pressure among the groups at any time (Supplemental Figure 1; supplemental material available online with this article; doi:10.1172/JCI90837DS1).

\section{Discussion}

After 6 weeks of refrigerated storage, with strict adherence to current FDA standards, transfusions of healthy adult volunteers with single, autologous, leukoreduced units of packed red cells over-
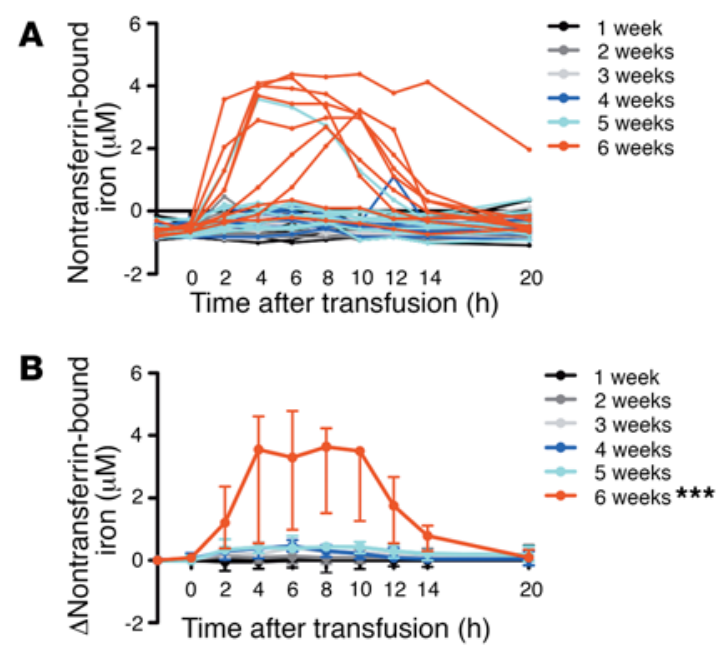

Figure 2. Nontransferrin-bound iron is increased predominantly after 6 weeks of red blood cell storage. (A) Circulating nontransferrin-bound iron levels from pretransfusion to all posttransfusion time points are shown for each subject who completed the study. Nontransferrin-bound iron levels are negative in healthy subjects using the ultrafiltration assay (39). (B) The medians (I bars represent interquartile ranges) are shown for the change in nontransferrin-bound iron from pretransfusion to all time points between 0 and 20 hours after transfusion. Statistical significance for ANOVA using post-hoc Tukey's test of the AUC among groups is shown. ${ }^{* *} P<0.001$ compared with all other groups. $n=52$ total. 
A
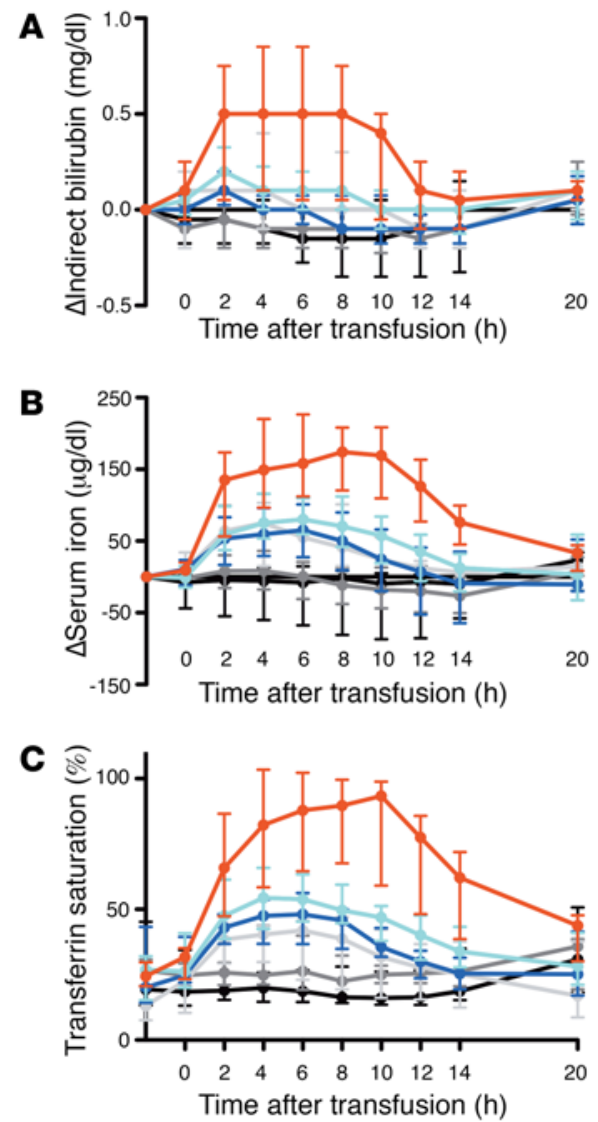

Figure 3. Markers of extravascular hemolysis are increased following transfusion of red cells stored for longer duration. (A and B) Medians (I bars represent interquartile ranges) for the changes in indirect bilirubin and serum iron, as labeled, are shown from pretransfusion to all posttransfusion time points. (C) Median transferrin saturation is also shown from pretransfusion to all posttransfusion time points. Statistical significance for ANOVA using post-hoc Tukey's test of the AUC among the groups is shown. $n=52$ total. ${ }^{*} P<0.05$; ${ }^{* *} P<0.01$; ${ }^{* *} P<0.001$. Asterisks without brackets represent significance compared with all other groups.

whelmed their physiologic capacity to process the cleared storage-damaged red cells, releasing large amounts of iron into the circulation. Transfusion effectiveness, in terms of increasing the hematocrit and the number of circulating red cells (i.e., posttransfusion red cell recovery), decreased with increasing storage time. Although indirect bilirubin, iron, ferritin, hepcidin, and transferrin saturation levels progressively increased in recipients in proportion to increasing storage duration, volunteers transfused with autologous red cells stored for 1 to 5 weeks did not produce significant amounts of circulating nontransferrin-bound iron, with the exception of 1 volunteer who received a 5-week-stored unit. In contrast, $78 \%$ (i.e., 7/9) of volunteers transfused with 6-weekstored red cells developed substantial amounts of circulating nontransferrin-bound iron that persisted for 10 hours or more. These results provide evidence of physiologic differences in the consequences of transfusing red cells stored for 6 weeks, as compared with those stored for 1 to 5 weeks.

The observed timing and pattern of laboratory results demonstrate extravascular hemolysis (i.e., phagocytosis of red cells by the monocyte/macrophage system in the liver and spleen of the recipient) of a subpopulation of storage-damaged, transfused red cells following storage for greater than 2 weeks. Indirect bilirubin and iron levels progressively increased with increasing storage time, without significant effects on lactate dehydrogenase, haptoglobin, or plasma-free hemoglobin concentrations. According to US FDA guidelines, up to $1 \%$ of ex vivo hemolysis is permissible in the storage bag prior to transfusion (2), which could lead to elevated plasma-free hemoglobin levels in some transfusion recipients. However, we found no differences in several markers of intravascular hemolysis (e.g., plasma-free hemoglobin, haptoglobin, and lactate dehydrogenase) in healthy subjects after transfusion of a single unit stored for 1 to 6 weeks. Thus, the circulating iron and indirect bilirubin levels observed after transfusion are almost certainly the catabolic breakdown products of the hemoglobin delivered to the monocyte/macrophage system by extravascular hemolysis.

In healthy adults, approximately $1 \mathrm{mg}$ of iron is released per hour from catabolism of senescent red cells (15). In contrast, current FDA criteria include an average 51-chromium posttransfusion red cell recovery of $75 \%$ or more in healthy volunteers at outdate (16), allowing up to $25 \%$ of transfused red cells to be cleared from the circulation in the first 24 hours, which is equivalent to approximately $60 \mathrm{mg}$ of iron (17). Because most storage-damaged red cells are cleared within the first hour after transfusion (18), the rate of macrophage iron export to plasma can exceed the rate of iron acquisition by circulating transferrin, resulting in the appearance of nontransferrin-bound iron. Thus, our results provide compelling evidence that transfusions of 6-week-stored units overwhelm the physiologic capacity of healthy volunteers to process the iron released from the catabolism of cleared, storage-damaged red cells.

Circulating nontransferrin-bound iron is the iron within the circulation that is neither bound to the physiologic iron-transport protein, transferrin, nor a constituent of heme or ferritin (19). In healthy individuals, little, if any, nontransferrin-bound iron is ever present within the circulation. In health, iron enters the plasma principally from the recycling of iron derived from senescent erythrocytes by a dedicated population of macrophages in the spleen, liver, and bone marrow (20). Much smaller amounts enter plasma from iron absorption by duodenal enterocytes or from mobilization of stores within hepatocytes. For all these sources, ferroportin, the cellular iron export protein, is the sole known conduit for iron to enter plasma. Ferroxidase activity is required for iron export through ferroportin, provided by ceruloplasmin in macrophages and by hephaestin in duodenal enterocytes. The release of iron into plasma does not require unsaturated transferrin; unsaturated transferrin does not enter cells, but accepts iron only after the exit of ferrous iron through ferroportin and its subsequent oxidation. Thus, iron is exported from cells via ferroportin whether or not circulating unsaturated transferrin is available. If the rate of iron influx into plasma exceeds the rate of iron acquisition by plasma transferrin, circulating nontransferrin-bound iron develops as a heterogeneous assortment of iron complexes (e.g., complexes of albumin, citrate, and potentially acetate, malate, or phosphate) (21). Thus, if the rate of iron efflux is sufficiently rapid, nontransferrin-bound iron is measurable at a transferrin saturation of approximately $70 \%$ and above (22). To control the efflux of iron into the circulation, ferroportin is regulated by hepcidin, which is itself regulated by iron, 

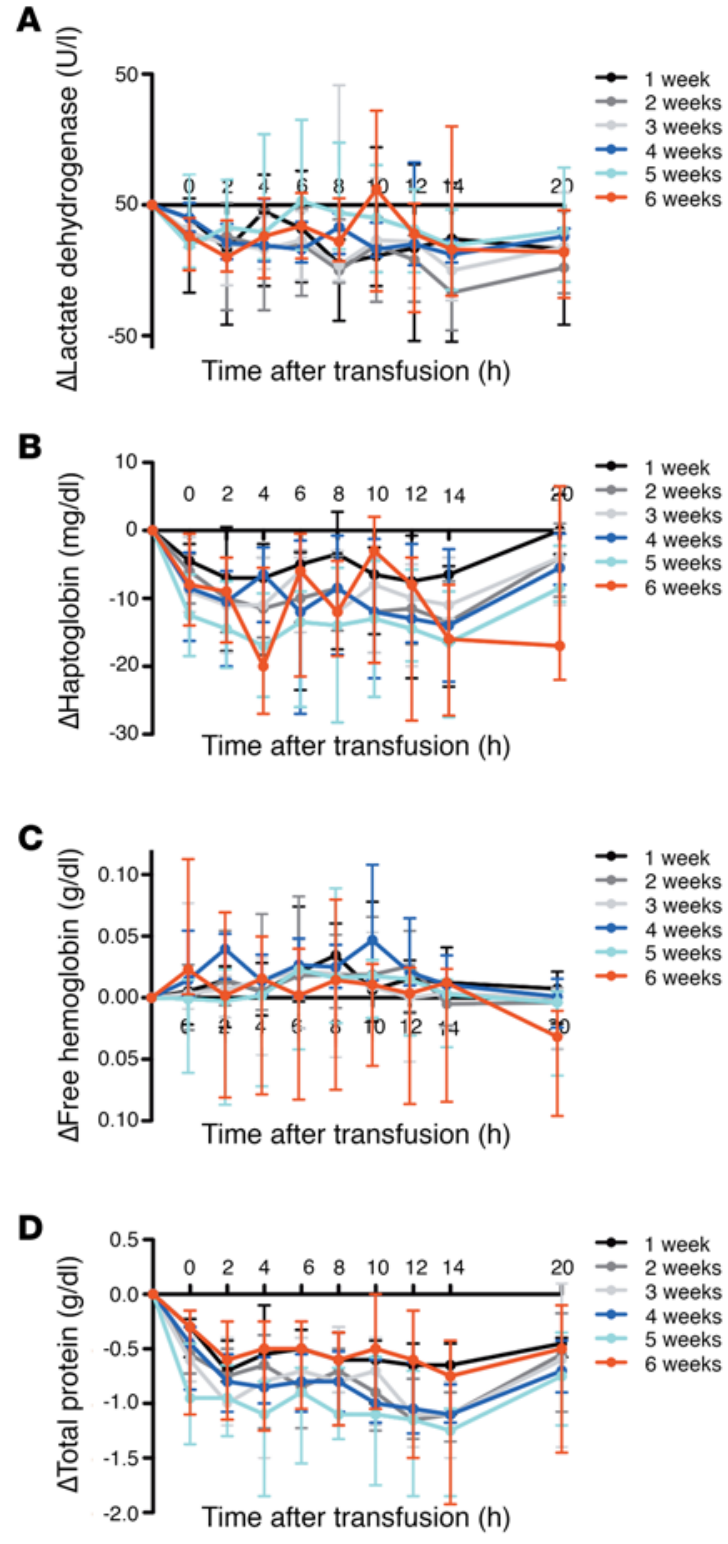

Figure 4. Markers of intravascular hemolysis are not increased following transfusion of a single, autologous red cell unit into healthy volunteers. (A-D) Medians (I bars represent interquartile ranges) for the changes in lactate dehydrogenase, haptoglobin, plasma-free hemoglobin, and total protein are shown from pretransfusion to all posttransfusion time points, as labeled. $n=52$ total.

inflammation (e.g., IL-6), and erythropoietic drive $(14,23)$. We did not observe any temporally associated changes in circulating IL-6 levels, suggesting that the increased hepcidin levels observed after transfusion of 6-week-stored red cells was driven predominantly by increases in transferrin saturation. Still, the rate and magnitude of hepcidin production were not sufficient to prevent the appearance of circulating nontransferrin-bound iron from approximately 2 to 14 hours after transfusion of 6-week-stored red cells.

Accumulating evidence indicates that the diverse mixture of iron complexes composed of nontransferrin-bound iron can directly produce harm (17). In the healthy volunteers in our study, with normal hepatic, splenic, and renal function and intact antioxidant defenses, no adverse events were observed during the approximately 10 hours of persistent circulation of nontransferrin-bound iron. Nonetheless, in animal studies, even short duration of circulating nontransferrin-bound iron enhanced the virulence of bloodstream pathogens (24-27). In addition, in a prior healthy human volunteer study, increased nontransferrin-bound iron in the sera obtained after transfusing 6-week-stored red cells enhanced proliferation in vitro of a pathogenic strain of E. coli (28). Similarly, increased serum iron present following oral iron supplementation enhanced proliferation in vitro of isolates of E. coli, Yersinia enterocolitica, Salmonella enterica serovar Typhimurium, and Staphylococcus epidermidis (29). Furthermore, in a retrospective analysis of patients receiving red cells stored for either 21 days or less or 35 days or more, there was a higher prevalence of infection in those receiving the older units $(P=0.007)(13)$. In other clinical settings, circulating nontransferrin-bound iron is also associated with increased risks of infectious complications; for example, following myelosuppression for acute leukemia, patients with plasma nontransferrin-bound iron levels greater than $2 \mu \mathrm{M}$ had a higher risk of Gram-negative sepsis $(P=0.0004)$ (30). Finally, by participating in Fenton chemistry, nontransferrin-bound iron causes oxidative damage and cytotoxicity and enhances endothelial expression of adhesion molecules, thereby increasing thrombotic risk $(19,21,31,32)$.

One limitation of the current study is that only 1 unit of packed red cells was transfused. Although hospitalized patients frequently receive multiple units of blood, the cumulative effect of transfusing multiple units was not examined. In addition, this study was performed in healthy, adult volunteers rather than in ill patients. However, according to current ethical standards, studies cannot randomize patients to transfusions restricted to the final week of storage $(4,12)$. Nonetheless, red cell recovery and hemolysis may be even worse in ill patients, as compared with the healthy volunteers (18). Indeed, in critically ill children and neonates, elevated circulating nontransferrin-bound iron levels were observed after transfusion, even before the final week of red cell storage $(33,34)$. Finally, for logistical reasons, 20-hour, as opposed to 24-hour, red cell recovery studies were performed. The 24-hour red cell recovery would be expected to be lower than the 20-hour recovery given the extra 4 hours of clearance. Nonetheless, the 20-hour red cell recovery is a close approximation to that after 24 hours because most clearance of storage-damaged red cells occurs during the first hour after transfusion (18).

In conclusion, the current results provide unequivocal evidence that the transfusion of red cells stored for 6 weeks exceeds the physiologic capacity of healthy volunteers to process the iron released from the catabolism of cleared red cells. Current FDA criteria allowing blood storage for 6 weeks are not founded on outcomes of clinical trials, but based on standards historically derived from expert opinion. In our study, the nontransferrin-bound iron levels measured in healthy volunteers after routine infusions of single units of autologous red cells are almost certainly lower than those that develop in ill patients, particularly after transfusion of multiple units of allogeneic red cells. Indeed, in observational studies of critically ill children and neonates, circulating nontransferrin-bound iron levels were elevated after transfusion $(33,34)$. Nonetheless, it is virtually impossible to conduct prospective clinical trials to 
A

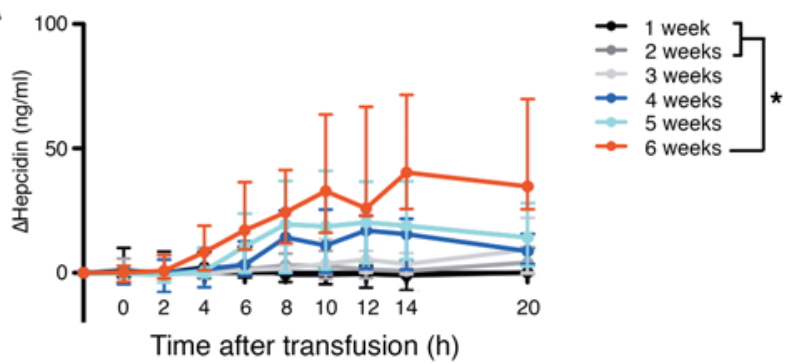

B

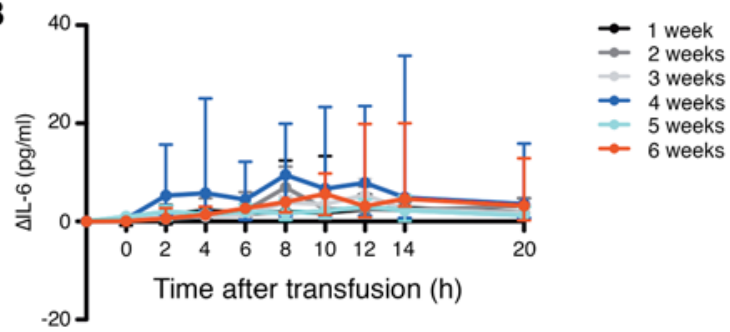

provide evidence-based guidance regarding the hazards posed by transfusing red blood cells stored for 35 to 42 days. Both the ethics of randomizing patients to transfusions restricted to the final week of storage and the logistics of enrolling the large numbers of patients needed to detect risks in specific subgroups are prohibitive. In addition, studies suggest that the blood supply would not be significantly affected by limiting the maximum allowable storage duration to 35 days (35-38); indeed, the United Kingdom, Ireland, the Netherlands, and the

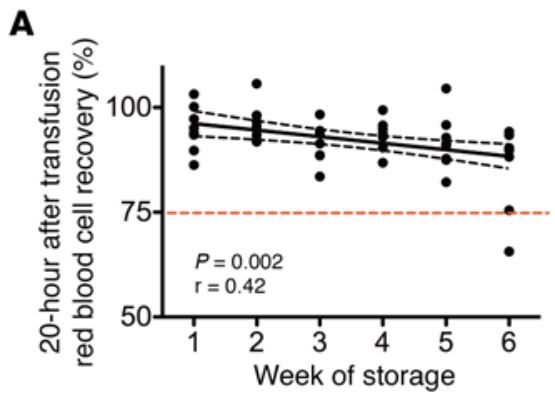

B

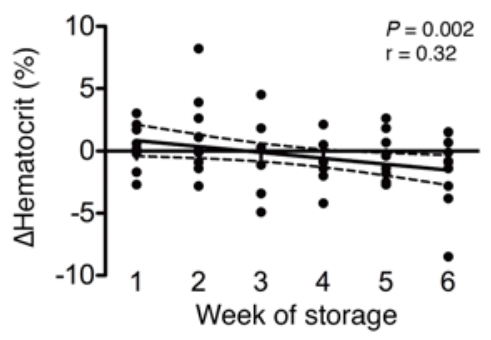

C

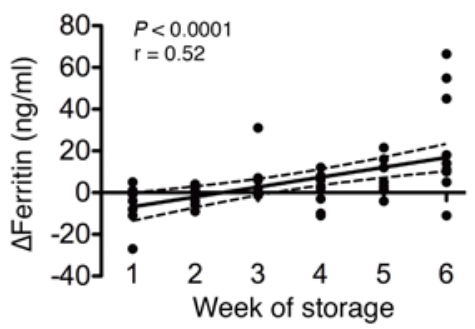

Figure 5. Serum hepcidin is increased following transfusion of red cells stored for longer duration, but this does not appear to be mediated by circulating IL-6 levels. (A and B) Medians (I bars represent interquartile ranges) for the changes in serum hepcidin and IL-6, as labeled, are shown from pretransfusion to all posttransfusion time points. Statistical significance for ANOVA using post-hoc Tukey's test of the AUC among the groups is shown. ${ }^{*} P<0.05 . n=52$ total.

NIH already have demonstrated the feasibility of this approach. Given the inability to perform definitive prospective randomized clinical trials examining red cell transfusions during the final week of storage, our evidence of the potential harm from extravascular hemolysis and circulating nontransferrin-bound iron, together with prudence and the precautionary principle, suggest that the maximal allowable storage period should be reduced to the minimum sustainable by the blood supply, with 35 days as an attainable goal.

\section{Methods}

Study design. A single center, parallel-group, randomized trial compared the effects of transfusing 1 autologous packed red cell unit after $1,2,3,4,5$, or 6 weeks of storage. The primary outcome was the AUC for the change in circulating nontransferrin-bound iron from pretransfusion to multiple time points after transfusion. Other iron-related parameters and hemolysis markers were secondary outcomes. Subjects and study coordinators were not blinded because subjects were scheduled for donation and transfusion based on the randomization scheme. Nonetheless, nonresearch staff nurses, without knowledge of study hypotheses, obtained all blood draws and blinded technologists performed all laboratory testing. A simple randomization scheme with 1 block was developed by the study statistician and recorded in consecutively numbered sealed paper slips by a nonstudy staff member. Following informed consent and screening for eligibility criteria, the next sequential paper slip was opened by the study coordinator to determine treatment allocation. Blood donations were at The New York Blood Center and transfusions at Columbia University Medical Center- New York Presbyterian Hospital. The first and last authors vouch for the completeness and accuracy of the data and analyses presented.

Participants and inclusion/exclusion criteria. Participants were principally recruited by flyers posted throughout the Columbia University Medical Center. Respondents were screened in person, and screening blood samples were collected to determine eligibility; criteria included age of 18 to 65 and meeting the standards for allogeneic blood donation (hemoglobin $>12.5 \mathrm{~g} / \mathrm{dl}$ and body weight $>110 \mathrm{lbs}$ [50 kg]).

Exclusion criteria were ineligibility for donation based on The New York Blood Center autologous blood donor questionnaire, sys-

Figure 6. Transfusion of red cells after longer duration of storage is associated with decreased red cell recovery, decreased change in hematocrit, and increased iron stores. (A) The 51-chromium 20-hour posttransfusion red blood cell recovery is shown. The dotted red line denotes the FDA criterion for acceptability (i.e., at outdate, on average $>75 \%$ of transfused red blood cells should still be circulating after 24 hours). $n=51$ total. (B and C) The changes in hematocrit and serum ferritin, respectively, for each subject from pretransfusion to 20 hours after transfusion are shown. $n=52$ total. Results of linear regression are shown with the $95 \% \mathrm{Cl}$ in dashed black lines. 
tolic blood pressure greater than 180 or less than $90 \mathrm{mmHg}$, diastolic blood pressure greater than 100 or less than $50 \mathrm{~mm} \mathrm{Hg}$, heart rate less than 50 or greater than 100 beats per minute, temperature greater than $99.5^{\circ} \mathrm{F}\left(>37.5^{\circ} \mathrm{C}\right)$ before donation, temperature greater than $100.4^{\circ} \mathrm{F}\left(>38^{\circ} \mathrm{C}\right)$ or subjective feeling of illness before transfusion (to avoid having a concurrent illness affect posttransfusion measurements), positive results on standard blood donor infectious disease testing, and positive urine pregnancy test for females.

Procedures. Participants were randomly assigned, in a 1:1:1:1:1:1 ratio, for a single autologous red cell transfusion after 1, 2, 3, 4, 5, or 6 weeks ( \pm 2 days) of storage. Each donated whole blood unit was leukoreduced and processed into a standard, packed, red cell unit in Additive Solution Formula 3 (AS-3; Haemonetics Corp.) by The New York Blood Center, shipped to the Columbia University Medical Center - New York Presbyterian Hospital Blood Bank, and refrigerator stored until issue. Immediately before issue, a $25 \mathrm{ml}$ aliquot, obtained from the unit using a sterile docking device, was radiolabeled with 51-chromium, as described (39).

Study subjects were admitted to the Columbia University Medical Center for 24 hours. After pretransfusion blood samples were collected, subjects were transfused at $200 \mathrm{ml}$ per hour. Vital signs were recorded before transfusion, 15 minutes after starting transfusion, and immediately and 1 hour after transfusion. Blood samples, obtained from a peripherally placed intravenous line, were collected immediately and at 2, 4, 6, 8, 10, 12, 14, and 20 hours after transfusion. Between 1 and 4 hours after transfusion, the 51-chromiumlabeled red cell aliquot was infused over 1 minute. Blood samples were then obtained every 2.5 minutes between 5 and 15 minutes after infusion and used to extrapolate the time zero and the final time point for chromium recovery (39).

Laboratory measures. Routine laboratory parameters were measured at the Columbia University Medical Center - New York Presbyterian Hospital Clinical Laboratories. Transferrin saturation and total iron binding capacity were calculated from the measured serum iron and unbound iron-binding capacity. When the unbound iron-binding capacity was below the clinical laboratory's reportable range, transferrin saturation was imputed by averaging the closest time points with calculable total iron-binding capacities. Nontransferrin-bound iron was measured using an ultrafiltration assay $(26,28)$; this method correlates well with other techniques (22). Plasma-free hemoglobin was measured by a modified cyanomethemoglobin method (40). Hepcidin and IL-6 were measured using commercial ELISA kits (Bachem and R\&D Systems, respectively), following the manufacturer's instructions.

Statistics. Between-group differences in binary and categorical variables were compared using Fisher's exact tests. Continuous variables were compared using Kruskal-Wallis tests. Data on the primary outcome of nontransferrin-bound iron and other secondary markers of hemolysis (e.g., indirect bilirubin, serum iron, transferrin saturation, hepcidin, lactate dehydrogenase, haptoglobin, free hemoglobin) were compared using an ANOVA with post-hoc Tukey's test of the AUC from the pretransfusion value through all time points up to 20 hours after transfusion. When calculating the AUC, peaks that were less than $10 \%$ of the distance from minimum to maximum were ignored. Pearson's linear regression was also performed to examine linear trends in AUC values and relationships between storage duration and change in hematocrit, posttransfusion red cell recovery, and ferritin level at 20 hours after transfusion, as compared with the pretransfusion levels. Analyses were performed using SAS software, version 9.4 (SAS Institute), and Prism, version 5 (GraphPad Software Inc.). A $P$ value of less than 0.05 was considered significant. Figures with error bars show medians with the interquartile range.

The target sample size of 60 participants was intended to achieve $90 \%$ power at $1 \% \alpha$ and a 1 -way analysis of covariance to test the between-group differences of 6 red cell storage duration groups of the AUC values for nontransferrin-bound iron at one-quarter of the range observed in a preliminary study (28). This allowed for up to a $20 \%$ dropout rate (i.e., only 8 subjects required per group).

Study approval. The study was conducted according to the Declaration of Helsinki and in accordance with good clinical practice guidelines. The study was approved by the Columbia University Medical Center and New York Blood Center Institutional Review Boards. All research participants provided written, informed consent prior to study participation. An independent DSMB at Columbia University Medical Center conducted regular safety reviews.

\section{Author contributions}

EAH, SLS, and GMB designed and EH and FR led the clinical trial. FR, SB, FLC, CL, AR, BSW, JN, HW, and ROF assisted with data collection. SS and AE assisted with the design of experiments. MS, $\mathrm{RY}$, and CD assisted with the radioactive chromium recovery studies. EAH and DJM analyzed the clinical data. YZG and BHS assisted with blood donation procedures. JS assisted with blood banking processes. FR and EAH drafted the first version of the manuscript. All authors edited the manuscript.

\section{Acknowledgments}

This work was supported by NIH grant HL115557 and by the National Center for Advancing Translational Sciences, NIH, through grant number UL1 TR000040. The content is solely the responsibility of the authors and does not necessarily represent the official views of the NIH. The authors thank the inpatient and outpatient nurses and staff of the Irving Center for Clinical and Translational Research for their outstanding patient care and support of our study. We also thank the human volunteers who took part in this study and Simone Glynn and Traci Mondoro for their support of transfusion medicine research.

Address correspondence to: Eldad A. Hod, Department of Pathology and Cell Biology, 630 West 168th St, Room P\&S 14-434, Laboratory of Transfusion Biology, College of Physicians \& Surgeons of Columbia University, New York, New York 10032, USA. Phone: 212.342.5648; E-mail: eh2217@cumc.columbia.edu.
1. Pfuntner A, Wier LM, Stocks C. Healthcare Cost and Utilization Project (HCUP) Statistical Briefs. Agency for Healthcare Research and Quality, Rockville, MD. http://www.hcup-us.ahrq.gov/ reports/statbriefs/sb162.pdf. Published September 2013. Accessed November 3, 2016.

2. Hess JR, Biomedical Excellence for Safer Transfusion (BEST) Collaborative. Scientific problems in the regulation of red blood cell products. Transfusion. 2012;52(8):1827-1835.

3. Zimring JC. Established and theoretical factors to consider in assessing the red cell storage lesion. 
Blood. 2015;125(14):2185-2190.

4. Remy KE, et al. Transfusion of recently donated (fresh) red blood cells (RBCs) does not improve survival in comparison with current practice, while safety of the oldest stored units is yet to be established: a meta-analysis. Vox Sang. 2016;111(1):43-54.

5. Fergusson DA, et al. Effect of fresh red blood cell transfusions on clinical outcomes in premature, very low-birth-weight infants: the ARIPI randomized trial. JAMA. 2012;308(14):1443-1451.

6. Dhabangi A, et al. Effect of transfusion of red blood cells with longer vs shorter storage duration on elevated blood lactate levels in children with severe anemia: the TOTAL randomized clinical trial. JAMA. 2015;314(23):2514-2523.

7. Steiner ME, et al. Effects of red-cell storage duration on patients undergoing cardiac surgery. N Engl JMed. 2015;372(15):1419-1429.

8. Lacroix J, et al. Age of transfused blood in critically ill adults. N Engl J Med. 2015;372(15):1410-1418

9. Whitaker B, Rajbhandary S, Kleinman S, Harris A, Kamani N. Trends in United States blood collection and transfusion: results from the 2013 AABB Blood Collection, Utilization, and Patient Blood Management Survey. Transfusion. 2016;56(9):2173-2183.

10. Glynn SA, Klein HG, Ness PM. The red blood cell storage lesion: the end of the beginning. Transfusion. 2016;56(6):1462-1468.

11. Flegel WA, Natanson C, Klein HG. Does prolonged storage of red blood cells cause harm? Br JHaematol. 2014;165(1):3-16.

12. Klein HG, Cortés-Puch I, Natanson C. More on the Age of Transfused Red Cells. N Engl JMed. 2015;373(3):283.

13. Goel R, et al. Red blood cells stored 35 days or more are associated with adverse outcomes in high-risk patients. Transfusion. 2016;56(7):1690-1698.

14. Nemeth E, Ganz T. Regulation of iron metabolism by hepcidin. Annu Rev Nutr. 2006;26:323-342.

15. Cook JD, Marsaglia G, Eschbach JW, Funk DD, Finch CA. Ferrokinetics: a biologic model for plasma iron exchange in man. JClin Invest. 1970;49(2):197-205.

16. Dumont LJ, AuBuchon JP. Evaluation of pro- posed FDA criteria for the evaluation of radiolabeled red cell recovery trials. Transfusion. 2008;48(6):1053-1060.

17. Hod EA, Spitalnik SL. Stored red blood cell transfusions: Iron, inflammation, immunity, and infection. Transfus Clin Biol. 2012;19(3):84-89.

18. Luten M, Roerdinkholder-Stoelwinder B, Schaap NP, de Grip WJ, Bos HJ, Bosman GJ. Survival of red blood cells after transfusion: a comparison between red cells concentrates of different storage periods. Transfusion. 2008;48(7):1478-1485.

19. Brissot P, Ropert M, Le Lan C, Loréal O. Non-transferrin bound iron: a key role in iron overload and iron toxicity. Biochim Biophys Acta. 2012;1820(3):403-410.

20. Andrews NC. Forging a field: the golden age of iron biology. Blood. 2008;112(2):219-230.

21. Hider RC. Nature of nontransferrin-bound iron. Eur J Clin Invest. 2002;32 Suppl 1:50-54.

22. de Swart L, et al. Second international round robin for the quantification of serum nontransferrin-bound iron and labile plasma iron in patients with iron-overload disorders. Haematologica. 2016;101(1):38-45.

23. Ganz T. Hepcidin and iron regulation, 10 years later. Blood. 2011;117(17):4425-4433.

24. Cortés-Puch I, et al. In a canine pneumonia model of exchange transfusion, altering the age but not the volume of older red blood cells markedly alters outcome. Transfusion. 2015;55(11):2564-2575.

25. Solomon SB, et al. Mortality increases after massive exchange transfusion with older stored blood in canines with experimental pneumonia. Blood. 2013;121(9):1663-1672.

26. Hod EA, et al. Transfusion of red blood cells after prolonged storage produces harmful effects that are mediated by iron and inflammation. Blood. 2010;115(21):4284-4292.

27. Prestia K, et al. Transfusion of stored blood impairs host defenses against Gram-negative pathogens in mice. Transfusion. 2014;54(11):2842-2851.

28. Hod EA, et al. Transfusion of human volunteers with older, stored red blood cells produces extravascular hemolysis and circulating non-transferrin-bound iron. Blood. 2011;118(25):6675-6682.
29. Cross JH, et al. Oral iron acutely elevates bacterial growth in human serum. Sci Rep. 2015;5:16670.

30. Belotti A, et al. Non transferrin bound iron (NTBI) in acute leukemias throughout conventional intensive chemotherapy: kinetics of its appearance and potential predictive role in infectious complications. Leuk Res. 2015;39(1):88-91.

31. Hershko C. Mechanism of iron toxicity. Food Nutr Bull. 2007;28(4 Suppl):S500-S509.

32. Mollet IG, et al. Low dose iron treatments induce a dna damage response in human endothelial cells within minutes. PLoS One. 2016;11(2):e0147990.

33. Stark MJ, Keir AK, Andersen CC. Does non-transferrin bound iron contribute to transfusion related immune-modulation in preterms? Arch Dis Child Fetal Neonatal Ed.2013;98(5):F424-F429.

34. L'Acqua C, et al. Red blood cell transfusion is associated with increased hemolysis and an acute phase response in a subset of critically ill children. Am J Hematol. 2015;90(10):915-920.

35. Dzik WH, et al. Factors affecting red blood cell storage age at the time of transfusion. Transfusion. 2013;53(12):3110-3119.

36. Blake JT, Hardy M, Delage G, Myhal G. Déjà-vu all over again: using simulation to evaluate the impact of shorter shelf life for red blood cells at Héma-Québec. Transfusion. 2013;53(7):1544-1558.

37. Fontaine MJ, Chung YT, Erhun F, Goodnough LT. Age of blood as a limitation for transfusion: potential impact on blood inventory and availability. Transfusion. 2010;50(10):2233-2239.

38. Grasas A, Pereira A, Bosch MA, Ortiz P, Puig L. Feasibility of reducing the maximum shelf life of red blood cells stored in additive solution: a dynamic simulation study involving a large regional blood system. Vox Sang. 2015;108(3):233-242.

39. Moroff G, Sohmer PR, Button LN. Proposed standardization of methods for determining the 24-hour survival of stored red cells. Transfusion. 1984;24(2):109-114.

40. Moore GL, Ledford ME, Merydith A. A micromodification of the Drabkin hemoglobin assay for measuring plasma hemoglobin in the range of 5 to 2000 mg/dl. Biochem Med. 1981;26(2):167-173. 